Acta Crystallographica Section E

Structure Reports

Online

ISSN 1600-5368

\section{6-Fluoro-1 $\mathrm{H}$-indole-3-carboxylic acid}

\section{Ming Lou* and Yang-Hui Luo}

College of Chemistry and Chemical Engineering, Southeast University, Nanjing 211189, People's Republic of China

Correspondence e-mail: chmsunbw@seu.edu.cn

Received 2 December 2011; accepted 18 April 2012

Key indicators: single-crystal X-ray study; $T=293 \mathrm{~K}$; mean $\sigma(\mathrm{C}-\mathrm{C})=0.002 \AA$; $R$ factor $=0.043 ; w R$ factor $=0.115 ;$ data-to-parameter ratio $=13.8$.

In the title compound, $\mathrm{C}_{9} \mathrm{H}_{6} \mathrm{FNO}_{2}$, all the non- $\mathrm{H}$ atoms are approximately coplanar, the carboxy $\mathrm{O}$ atoms deviating by 0.0809 and $-0.1279 \AA$ from the indole plane. In the crystal, $\mathrm{O}-\mathrm{H}$... O hydrogen bonds link the molecules into dimers which are linked via $\mathrm{N}-\mathrm{H} \cdots \mathrm{O}$ hydrogen bonds and $\pi-\pi$ interactions [centroid-centroid distance $=3.680$ (2) A]

\section{Related literature}

For the origin of the material studied, see: Kunzer \& Wendt (2011). For a related structure, see: Luo et al. (2011).

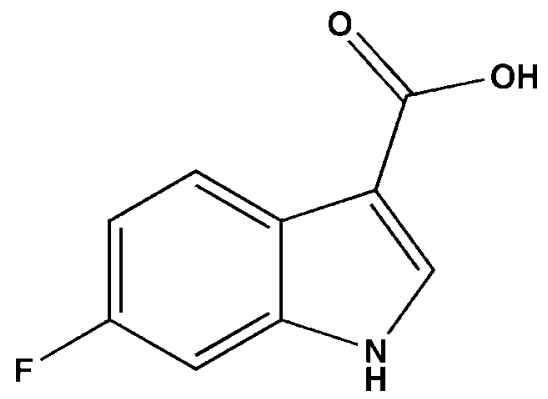

\section{Experimental}

\section{Crystal data}

$\mathrm{C}_{9} \mathrm{H}_{6} \mathrm{FNO}_{2} \quad M_{r}=179.15$
Monoclinic, $P 2_{1} / c$

$a=7.0054(14) \AA$

$b=11.699$ (2) $\AA$

$c=9.2947(19) \AA$

$\beta=104.15$ (3)

$V=738.7(3) \AA^{3}$

Data collection

Rigaku SCXmini diffractometer Absorption correction: multi-scan (CrystalClear; Rigaku, 2005)

$T_{\min }=0.961, T_{\max }=0.974$

Refinement

$R\left[F^{2}>2 \sigma\left(F^{2}\right)\right]=0.043$

$w R\left(F^{2}\right)=0.115$

$S=1.08$

1693 reflections

123 parameters
$Z=4$

Mo $K \alpha$ radiation

$\mu=0.13 \mathrm{~mm}^{-1}$

$T=293 \mathrm{~K}$

$0.3 \times 0.3 \times 0.2 \mathrm{~mm}$

Table 1

Hydrogen-bond geometry $\left(\AA{ }^{\circ}\right)$.

\begin{tabular}{lllll}
\hline$D-\mathrm{H} \cdots A$ & $D-\mathrm{H}$ & $\mathrm{H} \cdots A$ & $D \cdots A$ & $D-\mathrm{H} \cdots A$ \\
\hline $\mathrm{N} 1-\mathrm{H} 1 \cdots \mathrm{O} 1^{\mathrm{i}}$ & $0.86(2)$ & $2.159(19)$ & $2.8925(17)$ & $142.8(17)$ \\
$\mathrm{O} 2-\mathrm{H} 2 \cdots{ }^{\mathrm{ii}}$ & 0.82 & 1.78 & $2.5954(17)$ & 170
\end{tabular}

Symmetry codes: (i) $x,-y+\frac{1}{2}, z+\frac{1}{2}$; (ii) $-x+1,-y,-z$.

Data collection: CrystalClear (Rigaku, 2005); cell refinement: CrystalClear; data reduction: CrystalClear; program(s) used to solve structure: SHELXS97 (Sheldrick, 2008); program(s) used to refine structure: SHELXL97 (Sheldrick, 2008); molecular graphics: DIAMOND (Brandenburg \& Putz, 2005); software used to prepare material for publication: SHELXL97.

LM thanks Southeast University, Jiangsu Province, PRC.

Supplementary data and figures for this paper are available from the IUCr electronic archives (Reference: RN2099).

\title{
References
}

Brandenburg, K. \& Putz, H. (2005). DIAMOND. Crystal Impact GbR, Bonn, Germany.

Kunzer, A. R. \& Wendt, M. D. (2011). Tetrahedron, 52, 1815-1818.

Luo, Y.-H., Qian, X.-M., Gao, G., Li, J.-F. \& Mao, S.-L. (2011). Acta Cryst. E67, $\mathrm{m} 172$.

Rigaku. (2005). CrystalClear. Rigaku Corporation, Tokyo, Japan.

Sheldrick, G. M. (2008). Acta Cryst. A64, 112-122. 


\section{supporting information}

Acta Cryst. (2012). E68, o1580 [doi:10.1107/S1600536812016935]

\section{6-Fluoro-1 $H$-indole-3-carboxylic acid}

\section{Ming Lou and Yang-Hui Luo}

\section{S1. Comment}

Indole-3-carboxylic acid and its derivatives are important chemical materials, because they are excellent auxins for plants ( Kunzer \& Wendt, 2011) and drug intermediates for many pharmaceutical products (Luo et al., 2011).

The molecular structure of the title compound is shown in Fig. 1. All the non-H atoms are approximately coplanar: the carboxy $\mathrm{O}$ atoms deviating by 0.0809 and $-0.1279 \AA$ from the indole plane..

In the crystal structure of the title compound, intermolecular $\mathrm{O}-\mathrm{H} \cdots \mathrm{O}$ hydrogen bonds linked the molecules into dimers and the dimers are linked via intermolecular $\mathrm{N}-\mathrm{H} \cdots \mathrm{O}$ hydrogen bonds and $\pi-\pi$ interactions [centroid-centroid distance $=3.680(2) \AA]$ (Fig. 2).

\section{S2. Experimental}

The title compound was purchased commercially from ChemFuture PharmaTech, Ltd (Jiangsu) and used as received without further purification. Crystals of it were obtained by slow evaporation of a methanol solution.

\section{S3. Refinement}

All $\mathrm{H}$ atoms attached to $\mathrm{C}, \mathrm{N}$ and $\mathrm{O}$ atoms were fixed geometrically and treated as riding with $\mathrm{C}-\mathrm{H}=0.93 \AA(\mathrm{CH}), \mathrm{O}-$ $\mathrm{H}=0.82 \AA$ and $\mathrm{N}-\mathrm{H}=0.86 \AA$ with $U_{\text {iso }}(\mathrm{H})=1.2 U_{\text {eq }}(\mathrm{CH}), U_{\text {iso }}(\mathrm{H})=1.35 U_{\text {eq }}(\mathrm{N})$ and $U_{\text {iso }}(\mathrm{H})=1.5 U_{\text {eq }}(\mathrm{O})$. 


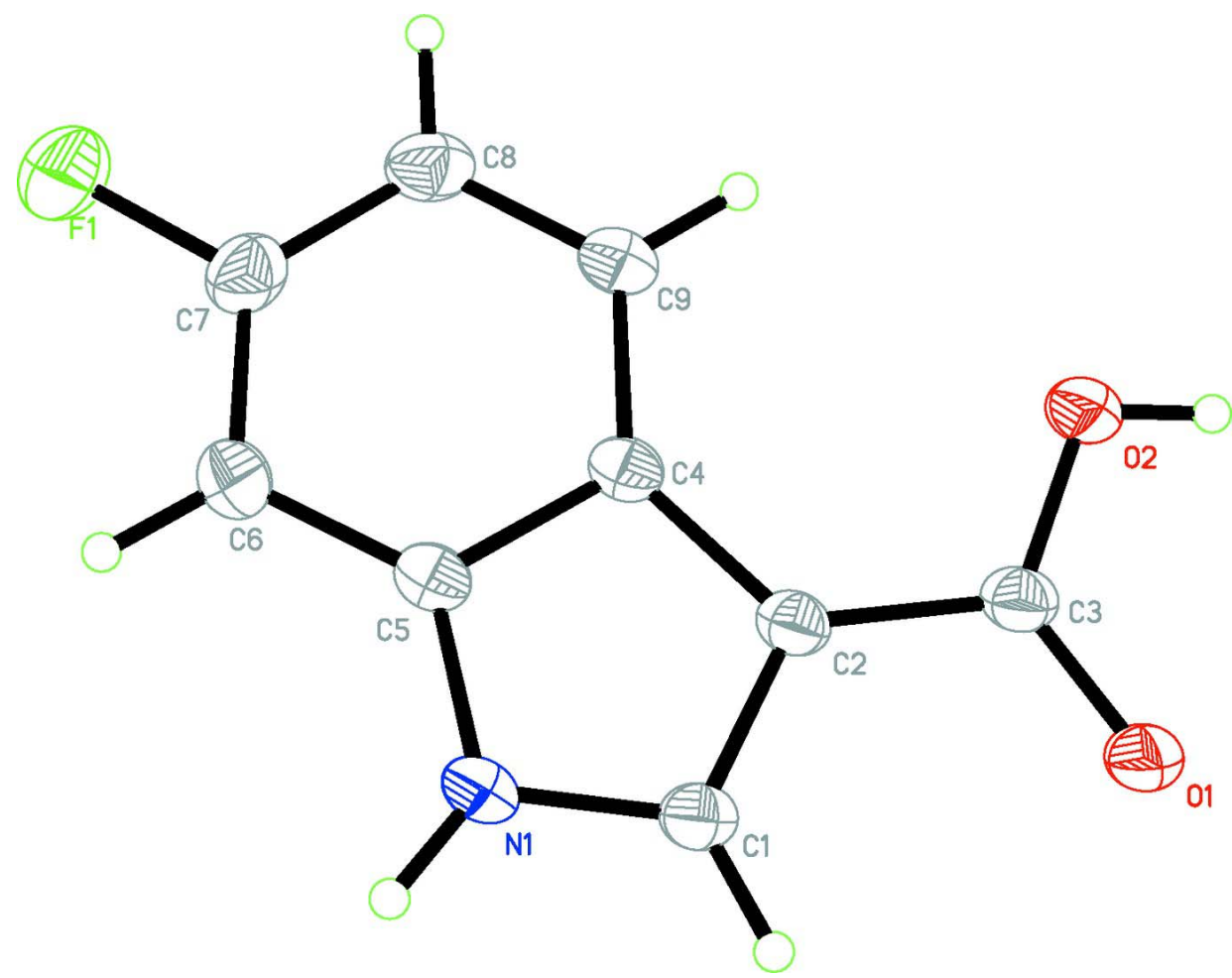

\section{Figure 1}

The molecular structure of the title compound with the atom-labelling scheme. Displacement ellipsoids are drawn at the $30 \%$ probability level. $\mathrm{H}$ atoms are represented as small spheres of arbitrary radii.

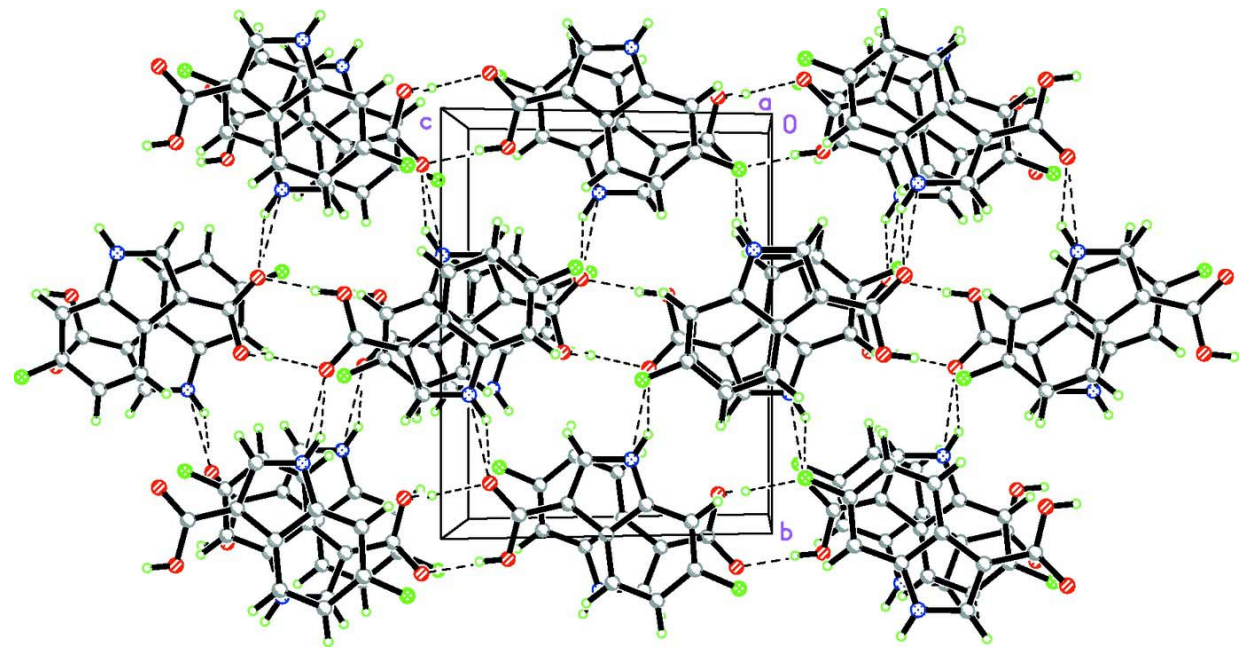

\section{Figure 2}

A packing view down the $a$ axis showing the three dimensional network. Intermolecular hydrogen bonds are shown as dashed lines. 


\section{6-Fluoro-1 $H$-indole-3-carboxylic acid}

Crystal data

$$
\begin{aligned}
& \mathrm{C}_{9} \mathrm{H}_{6} \mathrm{FNO}_{2} \\
& M_{r}=179.15 \\
& \text { Monoclinic, } P 2_{1} / c \\
& \text { Hall symbol: }-\mathrm{P} 2 \mathrm{ybc} \\
& a=7.0054(14) \AA \\
& b=11.699(2) \AA \\
& c=9.2947(19) \AA \\
& \beta=104.15(3)^{\circ} \\
& V=738.7(3) \AA^{3} \\
& Z=4
\end{aligned}
$$

\section{Data collection}

Rigaku SCXmini diffractometer

Radiation source: fine-focus sealed tube Graphite monochromator

Detector resolution: 13.6612 pixels $\mathrm{mm}^{-1}$

CCD_Profile_fitting scans

Absorption correction: multi-scan

(CrystalClear; Rigaku, 2005)

$T_{\min }=0.961, T_{\max }=0.974$

\section{Refinement}

Refinement on $F^{2}$

Least-squares matrix: full

$R\left[F^{2}>2 \sigma\left(F^{2}\right)\right]=0.043$

$w R\left(F^{2}\right)=0.115$

$S=1.08$

1693 reflections

123 parameters

0 restraints

Primary atom site location: structure-invariant direct methods
$F(000)=368$

$D_{\mathrm{x}}=1.611 \mathrm{Mg} \mathrm{m}^{-3}$

Mo $K \alpha$ radiation, $\lambda=0.71073 \AA$

Cell parameters from 1693 reflections

$\theta=3.5-27.5^{\circ}$

$\mu=0.13 \mathrm{~mm}^{-1}$

$T=293 \mathrm{~K}$

Block, brown

$0.3 \times 0.3 \times 0.2 \mathrm{~mm}$

7541 measured reflections

1693 independent reflections

1418 reflections with $I>2 \sigma(I)$

$R_{\text {int }}=0.033$

$\theta_{\max }=27.5^{\circ}, \theta_{\min }=3.5^{\circ}$

$h=-9 \rightarrow 9$

$k=-15 \rightarrow 15$

$l=-12 \rightarrow 12$

Secondary atom site location: difference Fourier map

Hydrogen site location: inferred from neighbouring sites

$\mathrm{H}$ atoms treated by a mixture of independent and constrained refinement

$w=1 /\left[\sigma^{2}\left(F_{\mathrm{o}}^{2}\right)+(0.0591 P)^{2}+0.1428 P\right]$ where $P=\left(F_{\mathrm{o}}{ }^{2}+2 F_{\mathrm{c}}{ }^{2}\right) / 3$

$(\Delta / \sigma)_{\max }<0.001$

$\Delta \rho_{\max }=0.21 \mathrm{e} \AA^{-3}$

$\Delta \rho_{\min }=-0.21 \mathrm{e} \AA^{-3}$

\section{Special details}

Geometry. All e.s.d.'s (except the e.s.d. in the dihedral angle between two 1.s. planes) are estimated using the full covariance matrix. The cell e.s.d.'s are taken into account individually in the estimation of e.s.d.'s in distances, angles and torsion angles; correlations between e.s.d.'s in cell parameters are only used when they are defined by crystal symmetry. An approximate (isotropic) treatment of cell e.s.d.'s is used for estimating e.s.d.'s involving 1.s. planes.

Refinement. Refinement of $F^{2}$ against ALL reflections. The weighted $R$-factor $w R$ and goodness of fit $S$ are based on $F^{2}$, conventional $R$-factors $R$ are based on $F$, with $F$ set to zero for negative $F^{2}$. The threshold expression of $F^{2}>\sigma\left(F^{2}\right)$ is used only for calculating $R$-factors(gt) $e t c$. and is not relevant to the choice of reflections for refinement. $R$-factors based on $F^{2}$ are statistically about twice as large as those based on $F$, and $R$ - factors based on ALL data will be even larger.

Fractional atomic coordinates and isotropic or equivalent isotropic displacement parameters $\left(\AA^{2}\right)$

\begin{tabular}{lllll}
\hline & $x$ & $y$ & $z$ & $U_{\text {iso }} * / U_{\text {eq }}$ \\
\hline O2 & $0.60596(19)$ & $-0.07115(8)$ & $0.16978(12)$ & $0.0489(3)$ \\
$\mathrm{H} 2$ & 0.5681 & -0.0785 & 0.0795 & $0.073^{*}$ \\
O1 & $0.5438(2)$ & $0.11097(9)$ & $0.10977(12)$ & $0.0514(3)$
\end{tabular}


supporting information

$\begin{array}{lllll}\text { F1 } & 0.96927(19) & -0.13190(10) & 0.89741(12) & 0.0727(4) \\ \text { N1 } & 0.70843(19) & 0.17044(11) & 0.55849(14) & 0.0394(3) \\ \text { C3 } & 0.5997(2) & 0.03550(12) & 0.20426(16) & 0.0377(3) \\ \text { C2 } & 0.6606(2) & 0.06339(11) & 0.35810(16) & 0.0343(3) \\ \text { C4 } & 0.74187(19) & -0.00793(11) & 0.48256(15) & 0.0330(3) \\ \text { C9 } & 0.7914(2) & -0.12279(12) & 0.50269(18) & 0.0397(4) \\ \text { H9 } & 0.7736 & -0.1718 & 0.4217 & 0.048^{*} \\ \text { C6 } & 0.8487(2) & 0.02441(13) & 0.74928(18) & 0.0432(4) \\ \text { H6 } & 0.8692 & 0.0722 & 0.8316 & 0.052^{*} \\ \text { C8 } & 0.8663(2) & -0.16262(13) & 0.6426(2) & 0.0465(4) \\ \text { H8 } & 0.8991 & -0.2394 & 0.6583 & 0.056^{*} \\ \text { C5 } & 0.7710(2) & 0.06329(12) & 0.60672(16) & 0.0351(3) \\ \text { C1 } & 0.6428(2) & 0.17026(12) & 0.41164(16) & 0.0382(3) \\ \text { H1A } & 0.5925 & 0.2335 & 0.3542 & 0.046^{*} \\ \text { C7 } & 0.8931(2) & -0.08866(15) & 0.76066(19) & 0.0475(4) \\ \text { H1 } & 0.702(3) & 0.2305(17) & 0.611(2) & 0.053(5)^{*}\end{array}$

Atomic displacement parameters $\left(\AA^{2}\right)$

\begin{tabular}{lllllll}
\hline & $U^{11}$ & $U^{22}$ & $U^{33}$ & $U^{12}$ & $U^{13}$ & $U^{23}$ \\
\hline O2 & $0.0787(8)$ & $0.0289(6)$ & $0.0382(6)$ & $-0.0018(5)$ & $0.0127(6)$ & $-0.0044(4)$ \\
O1 & $0.0856(9)$ & $0.0304(6)$ & $0.0364(6)$ & $-0.0084(5)$ & $0.0116(6)$ & $0.0019(4)$ \\
F1 & $0.0872(8)$ & $0.0638(8)$ & $0.0524(7)$ & $0.0055(6)$ & $-0.0111(6)$ & $0.0161(5)$ \\
N1 & $0.0514(7)$ & $0.0270(6)$ & $0.0399(7)$ & $-0.0006(5)$ & $0.0114(6)$ & $-0.0068(5)$ \\
C3 & $0.0479(8)$ & $0.0291(7)$ & $0.0388(8)$ & $-0.0050(6)$ & $0.0156(6)$ & $-0.0007(5)$ \\
C2 & $0.0397(7)$ & $0.0281(7)$ & $0.0370(8)$ & $-0.0039(5)$ & $0.0128(6)$ & $-0.0025(5)$ \\
C4 & $0.0321(6)$ & $0.0291(7)$ & $0.0393(8)$ & $-0.0030(5)$ & $0.0115(6)$ & $-0.0025(5)$ \\
C9 & $0.0417(8)$ & $0.0300(7)$ & $0.0475(9)$ & $0.0004(6)$ & $0.0114(7)$ & $-0.0042(6)$ \\
C6 & $0.0450(8)$ & $0.0430(8)$ & $0.0388(8)$ & $-0.0053(7)$ & $0.0046(6)$ & $-0.0044(6)$ \\
C8 & $0.0456(9)$ & $0.0329(8)$ & $0.0585(10)$ & $0.0055(6)$ & $0.0079(7)$ & $0.0050(7)$ \\
C5 & $0.0342(7)$ & $0.0306(7)$ & $0.0411(8)$ & $-0.0034(5)$ & $0.0101(6)$ & $-0.0038(6)$ \\
C1 & $0.0477(8)$ & $0.0287(7)$ & $0.0392(8)$ & $-0.0010(6)$ & $0.0124(6)$ & $0.0001(6)$ \\
C7 & $0.0442(8)$ & $0.0477(9)$ & $0.0447(9)$ & $0.0006(7)$ & $-0.0005(7)$ & $0.0089(7)$ \\
& & & & & & \\
\hline
\end{tabular}

Geometric parameters $\left(\AA,{ }^{\circ}\right)$

\begin{tabular}{llll}
\hline $\mathrm{O} 2-\mathrm{C} 3$ & $1.2916(17)$ & $\mathrm{C} 4-\mathrm{C} 9$ & $1.389(2)$ \\
$\mathrm{O} 2-\mathrm{H} 2$ & 0.8200 & $\mathrm{C} 4-\mathrm{C} 5$ & $1.3974(19)$ \\
$\mathrm{O} 1-\mathrm{C} 3$ & $1.2394(18)$ & $\mathrm{C} 9-\mathrm{C} 8$ & $1.360(2)$ \\
$\mathrm{F} 1-\mathrm{C} 7$ & $1.351(2)$ & $\mathrm{C} 9-\mathrm{H} 9$ & 0.9300 \\
$\mathrm{~N} 1-\mathrm{C} 1$ & $1.330(2)$ & $\mathrm{C} 6-\mathrm{C} 7$ & $1.357(2)$ \\
$\mathrm{N} 1-\mathrm{C} 5$ & $1.3668(19)$ & $\mathrm{C} 6-\mathrm{C} 5$ & $1.381(2)$ \\
$\mathrm{N} 1-\mathrm{H} 1$ & $0.86(2)$ & $\mathrm{C} 6-\mathrm{H} 6$ & 0.9300 \\
$\mathrm{C} 3-\mathrm{C} 2$ & $1.426(2)$ & $\mathrm{C} 8-\mathrm{C} 7$ & $1.373(3)$ \\
$\mathrm{C} 2-\mathrm{C} 1$ & $1.363(2)$ & $\mathrm{C} 8-\mathrm{H} 8$ & 0.9300 \\
$\mathrm{C} 2-\mathrm{C} 4$ & $1.427(2)$ & $\mathrm{C} 1-\mathrm{H} 1 \mathrm{~A}$ & 0.9300 \\
& & & $115.15(14)$
\end{tabular}




$\begin{array}{llll}\mathrm{C} 1-\mathrm{N} 1-\mathrm{C} 5 & 109.75(12) & \mathrm{C} 7-\mathrm{C} 6-\mathrm{H} 6 & 122.4 \\ \mathrm{C} 1-\mathrm{N} 1-\mathrm{H} 1 & 121.7(13) & \mathrm{C} 5-\mathrm{C} 6-\mathrm{H} 6 & 122.4 \\ \mathrm{C} 5-\mathrm{N} 1-\mathrm{H} 1 & 128.4(13) & \mathrm{C} 9-\mathrm{C} 8-\mathrm{C} 7 & 119.56(15) \\ \mathrm{O} 1-\mathrm{C} 3-\mathrm{O} 2 & 122.44(14) & \mathrm{C} 9-\mathrm{C} 8-\mathrm{H} 8 & 120.2 \\ \mathrm{O} 1-\mathrm{C} 3-\mathrm{C} 2 & 120.84(13) & \mathrm{C} 7-\mathrm{C} 8-\mathrm{H} 8 & 120.2 \\ \mathrm{O} 2-\mathrm{C} 3-\mathrm{C} 2 & 116.72(13) & \mathrm{N} 1-\mathrm{C} 5-\mathrm{C} 6 & 129.53(14) \\ \mathrm{C} 1-\mathrm{C} 2-\mathrm{C} 3 & 122.98(14) & \mathrm{N} 1-\mathrm{C} 5-\mathrm{C} 4 & 122.80(13) \\ \mathrm{C} 1-\mathrm{C} 2-\mathrm{C} 4 & 107.12(12) & \mathrm{C} 6-\mathrm{C} 5-\mathrm{C} 4 & 109.68(13) \\ \mathrm{C} 3-\mathrm{C} 2-\mathrm{C} 4 & 129.88(13) & \mathrm{N} 1-\mathrm{C} 1-\mathrm{C} 2 & 125.2 \\ \mathrm{C} 9-\mathrm{C} 4-\mathrm{C} 5 & 118.97(13) & \mathrm{N} 1-\mathrm{C} 1-\mathrm{H} 1 \mathrm{~A} & 125.2 \\ \mathrm{C} 9-\mathrm{C} 4-\mathrm{C} 2 & 135.39(13) & \mathrm{C} 2-\mathrm{C} 1-\mathrm{H} 1 \mathrm{~A} & 117.94(16) \\ \mathrm{C} 5-\mathrm{C} 4-\mathrm{C} 2 & 105.64(12) & \mathrm{F} 1-\mathrm{C} 7-\mathrm{C} 6 & 117.45(15) \\ \mathrm{C} 8-\mathrm{C} 9-\mathrm{C} 4 & 119.04(14) & \mathrm{F} 1-\mathrm{C} 7-\mathrm{C} 8 & 124.61(15) \\ \mathrm{C} 8-\mathrm{C} 9-\mathrm{H} 9 & 120.5 & \mathrm{C} 6-\mathrm{C} 7-\mathrm{C} 8 & \\ \mathrm{C} 4-\mathrm{C} 9-\mathrm{H} 9 & 120.5 & & \end{array}$

Hydrogen-bond geometry $\left(A,{ }^{\circ}\right)$

\begin{tabular}{lllll}
\hline$D-\mathrm{H} \cdots A$ & $D-\mathrm{H}$ & $\mathrm{H} \cdots A$ & $D \cdots A$ & $D-\mathrm{H} \cdots A$ \\
\hline $\mathrm{N} 1-\mathrm{H} 1 \cdots \mathrm{O} 1^{\mathrm{i}}$ & $0.86(2)$ & $2.159(19)$ & $2.8925(17)$ & $142.8(17)$ \\
$\mathrm{O} 2-\mathrm{H} 2 \cdots \mathrm{O} 1^{\mathrm{ii}}$ & 0.82 & 1.78 & $2.5954(17)$ & 170
\end{tabular}

Symmetry codes: (i) $x,-y+1 / 2, z+1 / 2$; (ii) $-x+1,-y,-z$. 